\title{
Monte Carlo Modeling of Radiative Heat Transfer in Particle-laden Flow
}

\author{
Erin Farbar*, Iain D. Boyd \\ Department of Aerospace Engineering, University of Michigan, Ann Arbor, USA, 48109 \\ Mahdi Esmaily-Moghadam \\ Department of Mechanical Engineering, Stanford University, Stanford, USA, 94305
}

\begin{abstract}
Three-dimensional numerical simulations are applied to model radiative heat transfer in a dispersed particle phase exhibiting preferential concentration typical of a turbulent, particle-laden flow environment. The dispersed phase is composed of micron-sized nickel particles, and the carrier phase is non-participating. The simulations are performed for a snapshot of the particle field using the Monte Carlo Ray Tracing method, and the spectral dependence of the optical properties is considered. Interaction between the particles and radiation is modeled by projecting the particle locations onto an Eulerian mesh. Results show that the optically thin approximation results in errors in predicted particle heat transfer of up to $35 \%$ at some locations in the particle field. Oxidation is shown to change the absorption efficiency of the particles significantly, while consideration of non-spherical particle shapes results in relatively small changes in the predicted optical properties of the particles.
\end{abstract}

Keywords: particle-laden flow, Monte Carlo, oxidation, particle shape

\section{Nomenclature}

a Deformation parameter for Chebyshev particles

\footnotetext{
* Corresponding author.

Email address: efarbar@umich.edu (Erin Farbar)
}

Preprint submitted to Journal of Quantitative Spectroscopy and Radiative TransferJune 17, 2016

(C) 2016. This manuscript version is made available under the Elsevier user license http://www.elsevier.com/open-access/userlicense/1.0/ 


\begin{tabular}{|c|c|}
\hline$A_{\mathrm{p}}$ & Projected area of particle, $\mathrm{m}^{2}$ \\
\hline$c_{\mathrm{O}}$ & Speed of light in a vacuum, $299792458 \mathrm{~m} / \mathrm{s}$ \\
\hline$C_{\mathrm{p}}$ & Air isobaric heat capacity, $1012 \mathrm{~J} / \mathrm{kg} / \mathrm{K}$ \\
\hline$C_{\mathrm{v}}$ & Air isochoric heat capacity, $723 \mathrm{~J} / \mathrm{kg} / \mathrm{K}$ \\
\hline$C_{\mathrm{a}}$ & Particle heat capacity, J/kg/K \\
\hline$D$ & Depth of domain, m \\
\hline$D_{\mathrm{t}}$ & Total distance traveled in a cell, $\mathrm{m}$ \\
\hline$E_{\mathrm{bb}}$ & Blackbody emissive power, $\mathrm{W} / \mathrm{m}^{2} / \mu \mathrm{m}$ \\
\hline$f_{\mathrm{p}}$ & Particle Stokes drag, $\mathrm{kgm} / \mathrm{s}^{2}$ \\
\hline$g$ & Phase function asymmetry parameter \\
\hline$g$ & Gravitational acceleration, $\mathrm{m} / \mathrm{s}^{2}$ \\
\hline$G_{\mathrm{i}}$ & Radiation intensity in wavelength bin $\mathrm{i}, \mathrm{W} / \mathrm{m}^{2} / \mu \mathrm{m}$ \\
\hline$G$ & Direction-averaged radiation intensity, $\mathrm{W} / \mathrm{m}^{2}$ \\
\hline$h$ & Planck's constant, $6.626070 \times 10^{-34} \mathrm{Js}$ \\
\hline$i$ & Imaginary unit, $\sqrt{-1}$ \\
\hline$I$ & Identity tensor \\
\hline$I_{1}$ & Intensity of the lamp source, $\mathrm{W} / \mathrm{m}^{2}$ \\
\hline$k$ & Air thermal conductivity, $\mathrm{W} / \mathrm{m} / \mathrm{K}$ \\
\hline$k_{\mathrm{B}}$ & Boltzmann's constant, $1.3807 \times 10^{-23} \mathrm{~J} / \mathrm{K}$ \\
\hline$L$ & Length of domain, $\mathrm{m}$ \\
\hline$m$ & Complex index of refraction, $\mathrm{m}=\mathrm{n}+\mathrm{ik}$ \\
\hline$m_{\mathrm{p}}$ & Particle mass, $\mathrm{kg}$ \\
\hline$n_{\mathrm{p}}$ & Particle number density, $\mathrm{m}^{-3}$ \\
\hline$N_{\eta}$ & Number of wavelength bins \\
\hline$N_{\mathrm{p}}$ & Number of particles in a grid cell \\
\hline$N_{\mathrm{r}}$ & Number of rays \\
\hline $\mathrm{Nu}$ & Particle Nusselt number, 2 \\
\hline$p$ & Air hydrodynamic pressure, $\mathrm{Pa}$ \\
\hline$P$ & Air thermodynamic pressure, $\mathrm{Pa}$ \\
\hline$P_{\mathrm{p}, \mathrm{i}}$ & Power of particle in wavelength bin i, W \\
\hline
\end{tabular}




\begin{tabular}{|c|c|}
\hline$P_{\mathrm{r}}$ & Power of ray, $\mathrm{W}$ \\
\hline$Q_{\text {abs }}$ & Absorption efficiency \\
\hline$Q_{\text {sca }}$ & Scattering efficiency \\
\hline$\dot{Q}_{\mathrm{p}}$ & Heat transfer rate to particle, $\mathrm{W}$ \\
\hline$r_{\mathrm{o}}$ & Unperturbed particle radius, $\mathrm{m}$ \\
\hline$r_{\mathrm{p}}$ & Particle radius, $\mathrm{m}$ \\
\hline$R$ & Ideal gas constant for air, $288 \mathrm{~J} / \mathrm{K}$ \\
\hline$s_{\mathrm{p}}$ & Particle to air heat transfer, $\mathrm{W}$ \\
\hline $\mathrm{St}_{\eta}$ & Particle Stokes number based on the Kolmogorov time scale \\
\hline $\mathrm{St}^{+}$ & Particle Stokes number based on the Viscous time scale \\
\hline$t$ & time, $\mathrm{s}$ \\
\hline$t_{\mathrm{ox}}$ & Thickness of oxide layer, $\mathrm{m}$ \\
\hline$T$ & Stress tensor, $\mathrm{Pa}$ \\
\hline$T$ & Air temperature, $\mathrm{K}$ \\
\hline$T_{\mathrm{p}}$ & Particle temperature, $\mathrm{K}$ \\
\hline$T_{1}$ & Temperature of the lamp source, $\mathrm{K}$ \\
\hline$T_{\mathrm{n}}$ & Chebyshev polynomial of the first kind and degree $n$ \\
\hline$u$ & Air velocity, m/s \\
\hline $\boldsymbol{u}_{\mathrm{g}}$ & Particle velocity, m/s \\
\hline$V_{\mathrm{c}}$ & Volume of grid cell, $\mathrm{m}^{-3}$ \\
\hline$W$ & Width of computational domain, m \\
\hline$x$ & Particle size parameter \\
\hline $\mathbf{x}_{\mathrm{p}}$ & Position vector of particle, $\mathrm{m}$ \\
\hline$\delta_{a}$ & percent difference of parameter a \\
\hline$\delta$ & Dirac delta function \\
\hline$\Delta x$ & Length of cell side in computational mesh, $\mathrm{m}$ \\
\hline$\epsilon$ & Emissivity \\
\hline$\varepsilon_{\mathrm{OT}}$ & Relative error in the optically thin approximation \\
\hline$\eta_{\mathrm{i}}$ & Wavelength bin i \\
\hline$\Delta \eta_{\mathrm{i}}$ & Width of wavelength bin $\mathrm{i}, \mathrm{m}^{-1}$ \\
\hline
\end{tabular}




$\begin{array}{ll}\mu & \text { Air dynamic viscosity, } \mathrm{kg} / \mathrm{m} / \mathrm{s} \\ \lambda & \text { Wavelength of radiation, } \mathrm{m} \\ \rho & \text { Reflectivity } \\ \rho_{\mathrm{a}} & \text { Air mass density, } \mathrm{kg} / \mathrm{m}^{3} \\ \rho_{\mathrm{p}} & \text { Particle mass density, } \mathrm{kg} / \mathrm{m}^{3} \\ \sigma_{\mathrm{abs}} & \text { Absorption coefficient, } \mathrm{m}^{-1} \\ \sigma_{\text {sca }} & \text { Scattering coefficient, } \mathrm{m}^{-1} \\ \tau & \text { Optical depth } \\ \tau_{\mathrm{p}} & \text { Particle relaxation time, } \mathrm{s}\end{array}$

\section{Introduction}

Irradiated flows containing a dispersed particle phase occur in many physical systems. Numerical techniques have been applied to simulate dispersed phase flows with applications to particle-based solar receivers [1 4] and reactors [5, 6], 5 combustion chambers [7, solid rocket motors and plumes [8, 9], atmospheric clouds [10, 11, and fire protection systems [12. Due to the complex nature of these physical systems, detailed studies of the three-way coupled interactions between the flow, radiation and particles are only just beginning to be reported in the literature [13.

In initial studies of this three-way coupled interaction, a simple model for the radiative transfer is used such that all particles receive the same incident radiative flux, and it is completely absorbed [13. In other words, the fluidparticle medium is treated as a perfectly absorbing, optically thin medium. With an eye towards fully-coupled simulations including detailed models for radiative

15 transfer to the particle phase, the current work focuses on the development of a Monte Carlo Ray Tracing (MCRT) code for modeling radiative transfer to a dispersed particle phase, and the development of the physical models required to simulate one such system that employs micron-scale metallic particles. The intent is to use this MCRT code in future studies to assess the accuracy of more 
computationally efficient methods for solving the Radiative Transfer Equation (RTE), such as the Discrete Ordinates Method (DOM), when they are applied to model radiative transfer through a preferentially concentrated particle phase.

A review of radiative properties of dispersed media and radiative transfer modeling strategies is given in [14], while a specific discussion of Monte 25 Carlo simulation strategies in participating media is given in [15]. The earliest attempts to model radiative transfer in a medium containing participating particles using MCRT are described in [16]. In more recent studies using the MCRT technique, multidimensionality is neglected [6], the numerical method relies on the presence of a steady-state particle distribution [8] or periodicity of the domain [5], or inhomogeneities in the spatial distribution of the particles are neglected [17]. Each of these limitations is addressed in the current work, which builds upon the methods previously presented in [8].

In the current study, the MCRT code and associated physical models are used to model radiative transport through a channel containing micron-scale nickel particles. The particles interact with the turbulent flow of air through the channel, leading to a preferentially concentrated particle field [18. The particle clustering introduces strong inhomogeneity in the particle concentration field and has direct implications in radiation transport modeling. The parameters of the channel, particles and air flow are chosen to represent a regime appropriate

40 for particle-based solar receivers used for energy harvesting and storage. One realization of particle positions is considered in the current study. The sensitivity of the predictions to the assumptions made during the development of the physical models is addressed.

The outline of the paper is as follows. In Section 2, a description of the 45 physical system addressed in this work is given, including the properties of the particulate medium under consideration. In Section 3 , details of the computational model for the fluid-particle behaviour, the MCRT code used to solve the RTE, and the method used to describe the optical properties of the discrete particles are given. Results of the Monte Carlo simulations are presented in 50 Section 4 including discussions of the error associated with the optically thin 
approximation. The validity of the geometric optics approximation, the effects of particle oxidation, and the effects of non-spherical particle shapes are also discussed in Section 4. Lastly, conclusions are drawn in Section 5.

\section{Description of the Particle-laden Flow Environment}

The engineering system modeled in this work is designed to represent operating conditions that may be created experimentally as a part of a larger research program. The apparatus consists of a vertical channel with observation windows covering a small portion of the channel, referred to as the test section. Particles are injected at the top of the channel, and are carried by air through the channel. The particle mass loading ratio is $10 \%$. Table 1 gives the flow conditions at the channel entrance, where the air is at room temperature. The nominal particle density is the density that would exist if the particles were uniformly distributed in the channel. The nominal optical thickness is calculated using an emissivity of 0.22 , which is the Planck-mean value for the particles and is calculated in Section 3.1

The physical parameters of this model system are selected in order to produce turbulent channel flow with a Stanton number that is on the order of unity. In this flow regime, regions of preferentially concentrated particles form [18]; in these regions, absorption of radiative energy is non-uniform. The result of this non-uniformity is temperature gradients in the flow, and potentially a reduction in overall efficiency of the system.

A simplified model of the irradiated portion of channel is considered in this work. The geometry of the computational domain is shown in Figure 1 . The height of the test section is $H=0.16 \mathrm{~m}$, the width is $W=0.04 \mathrm{~m}$, and the depth is $D=0.04 \mathrm{~m}$. The light source is modeled as an array of lamps that are assumed to emit as a blackbody with a temperature of $2500 \mathrm{~K}$ and a total power of $20 \mathrm{~kW}$. The emitted radiation is assumed to be perfectly collimated due to mirrors used to focus the radiation on the test section, and is directed along the $+\mathrm{Z}$ axis. All other surfaces are modeled as cold, black walls. 
Table 1: Flow conditions at the channel entrance.

\begin{tabular}{lr}
\hline \hline Parameter & Value \\
\hline air bulk velocity & $2 \mathrm{~m} / \mathrm{s}$ \\
air density, $\rho_{\mathrm{a}}$ & $1.2 \mathrm{~kg} / \mathrm{m}^{3}$ \\
air temperature, $T$ & $300 \mathrm{~K}$ \\
flow bulk Reynolds number & 5139 \\
flow friction Reynolds number & 343 \\
nominal particle number density, $n_{\mathrm{p}}$ & $1.94 \times 10^{10} \mathrm{~m}^{-3}$ \\
particle radius, $r_{\mathrm{p}}$ & $6.0 \times 10^{-6} \mathrm{~m}$ \\
particle mass density, $\rho_{\mathrm{p}}$ & $8900 \mathrm{~kg} / \mathrm{m}^{3}$ \\
particle heat capacity, $C_{\mathrm{p}}$ & $450 \mathrm{~J} / \mathrm{kg} / \mathrm{K}$ \\
particle Nusselt number, Nu & 2 \\
particle mass loading ratio $\left(n m_{\mathrm{p}} / \rho_{\mathrm{a}}\right)$ & $10 \%$ \\
nominal optical thickness $\left(n_{\mathrm{p}} \pi r_{\mathrm{p}}^{2} \epsilon D\right)$ & 0.02 \\
\hline \hline
\end{tabular}
particles are drawn in this figure, but there are approximately four million particles in the simulation domain. Contours of the number density of the particle field are constructed by projecting the particle locations onto an Eulerian mesh and computing the mean particle number density in each grid cell. The particle number density on a plane parallel to the incident radiation from the lamp source is shown in Figure 1 .

\section{Numerical Method}

The flow of the turbulent air and particles is considered in a separate computational model, using Direct Numerical Simulation of the Navier-Stokes equa90 tions coupled to Lagrangian tracking of point-particles. This portion of the work is described in detail in Section 3.2 . The input to the MCRT simulations 


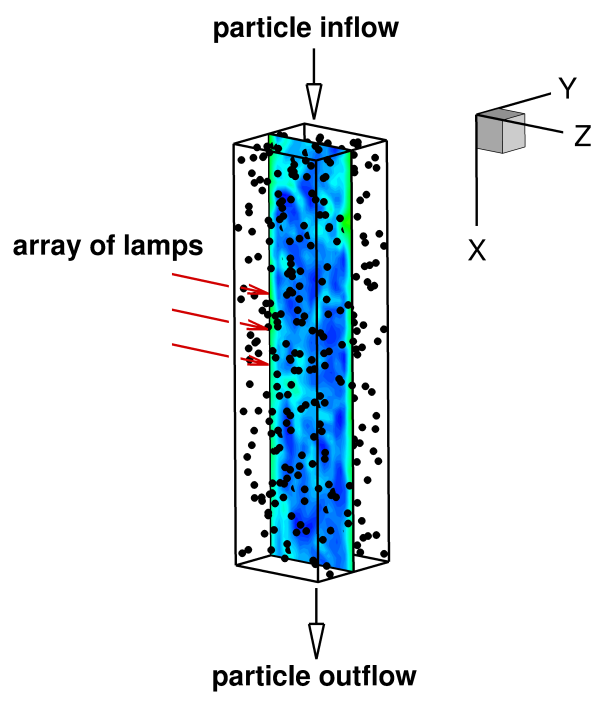

Figure 1: Geometry of the computational domain showing the location of the lamp source and particle inflow and outflow boundaries, a subset of the particle locations, and contours of particle number density in the center of the channel.

presented in this work is the particle field at one location in time; that is, a snap-shot of the particle-laden turbulent flow.

\subsection{Optical Properties}

The particles are nickel with a diameter of $12 \mu \mathrm{m}$. Nickel is selected in order to provide the highest absorption efficiency while simultaneously satisfying safety considerations for a future experimental facility, and producing Stanton numbers in the range of interest. The complex index of refraction for nickel is taken from [19], and is a function of the wavelength of incident radiation. The particles are assumed to be unoxidized, perfectly spherical, and of uniform diameter in the initial simulations. The first two assumptions are assessed in Section 4.

A band-averaged model for the optical properties of nickel particles is constructed using the geometric optics (GO) approximation. The use of this assumption permits diffraction of radiation to be separated from reflection and

refraction, and the absorption and scattering efficiencies are simply given by the 
emissivity, $\epsilon$, and reflectivity, $\rho$, of nickel. The projected area of a particle for absorption and reflection is equal to $A_{\mathrm{p}}=\pi r_{\mathrm{p}}^{2}$. The nominal particle properties reported in Section 2 give a size parameter of $\mathrm{x}=38$ at $\lambda=1 \mu \mathrm{m}$, where $\mathrm{x}$ is defined as

$$
x=\frac{2 \pi r_{\mathrm{p}}}{\lambda} .
$$
domain, ensuring the gas and particle statistics are captured realistically in the simulations. 


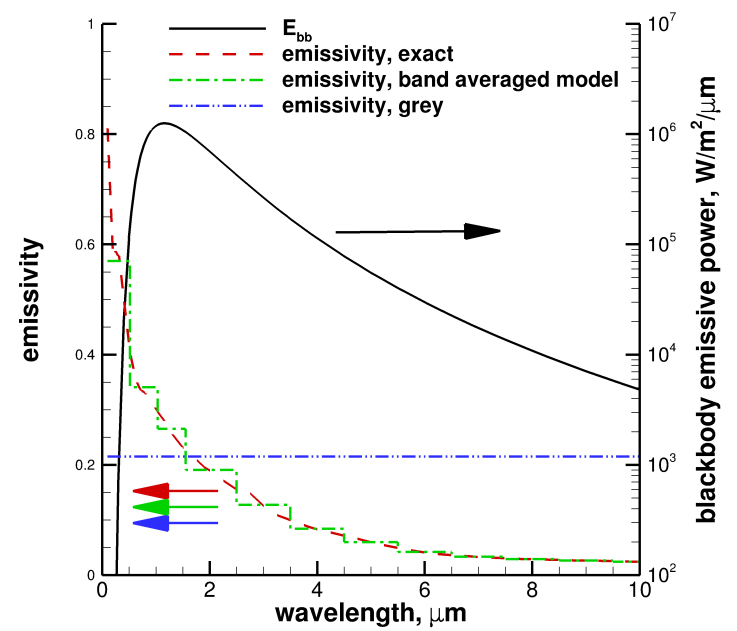

Figure 2: Emissivity of nickel particles as a function of wavelength, and Planck blackbody emissive power spectrum.

In a physically realistic scenario, fully-developed conditions are achieved using a long duct with an aspect ratio of order hundred. To avoid excessive computational cost of simulating a long duct with an inflow-outflow BC, we simulate a much shorter domain with a periodic BC (left domain in Figure 3. To ensure particle statistics are correctly resolved by the periodic BC, we performed an additional simulation with the inflow-outflow BC in a domain that has an aspect ratio of 144 (i.e. $5.76 \times 0.04 \times 0.4 \mathrm{~m}^{3}$ ). The result of this simulation showed that the conditions toward the outlet are homogeneous and similar to the periodic simulation. Therefore, to generate flow and particle concentration field at the inlet of the radiated domain, a concurrent periodic simulation is performed at which the gas temperature is constant and particles are exposed to no radiation. All of the flow conditions that are shown in Table1 are obtained from this domain.

In the second computational domain, a particle laden flow, which is exposed to homogeneous radiation, is simulated (right domain in Figure 3). The gas and particle conditions are extracted from the periodic simulations and imposed at 
Table 2: Properties of the band-averaged model for nickel.

\begin{tabular}{lr}
\hline \hline Wavelength Band, $\mu \mathrm{m}$ & Emissivity \\
\hline $0.00-0.50$ & 0.57 \\
$0.50-1.0$ & 0.34 \\
$1.0-1.5$ & 0.27 \\
$1.5-2.5$ & 0.19 \\
$2.5-3.5$ & 0.12 \\
$3.5-4.5$ & 0.084 \\
$4.5-5.5$ & 0.060 \\
$5.5-6.5$ & 0.042 \\
$6.5-7.5$ & 0.033 \\
$7.5-8.5$ & 0.029 \\
$8.5-9.5$ & 0.026 \\
$9.5-10.5$ & 0.024 \\
\hline \hline
\end{tabular}

the inlet of this domain. At the outlet, a zero Neumann BC is imposed. In this domain, the conditions are inhomogeneous in the streamwise direction due to the temperature variation. Due to the temperature increase, air expands toward the outlet, reducing the particle number density. As a result, $n_{\mathrm{p}}$ in this domain is $27 \%$ lower than the periodic domain.

Heat transfer from the particles changes the density of air. Hence, in the gas phase, the conservation of mass is satisfied by

$$
\frac{\partial}{\partial t} \rho_{a}+\nabla \cdot\left(\rho_{a} \boldsymbol{u}\right)=0
$$

and the conservation of momentum using

$$
\frac{\partial}{\partial t}\left(\rho_{a} \boldsymbol{u}\right)+\nabla \cdot\left(\rho_{a} \boldsymbol{u} \otimes \boldsymbol{u}\right)=\nabla \cdot \boldsymbol{T}+\rho_{a} \boldsymbol{g}+\sum_{p} \boldsymbol{f}_{p} \delta\left(\boldsymbol{x}-\boldsymbol{x}_{p}\right)
$$




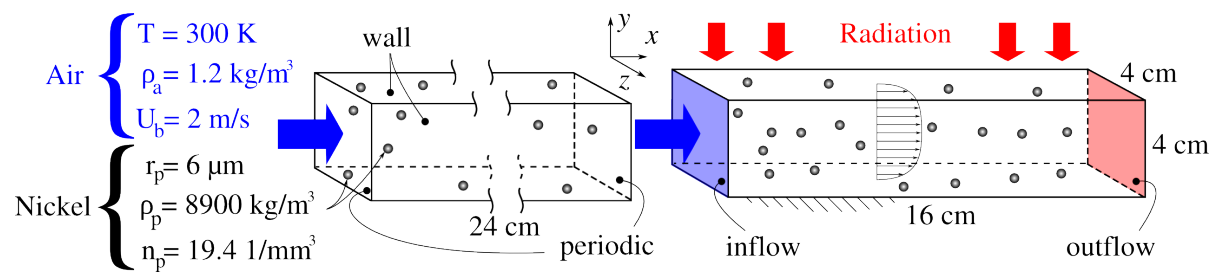

Figure 3: A schematic of the DNS set up. A particle-laden turbulent flow is simulated in two domains. An isothermal simulation is performed in the periodic domain (left) to generate an inflow condition for the simulation of the radiated domain (right).

in which

$$
\boldsymbol{T}=-p \boldsymbol{I}+\mu\left(\nabla \boldsymbol{u}+(\nabla \boldsymbol{u})^{\mathrm{T}}-\frac{2}{3} \nabla \cdot \boldsymbol{u I}\right)
$$

and $\boldsymbol{g}=(9.81,0,0) \mathrm{m} / \mathrm{s}^{2}, \boldsymbol{f}_{\mathrm{p}}$ is given in the following, and $\mu=\mu(T)$ is calculated based on the Sutherland's formula for air [21]. The last term in Equation 3. which involves a summation over all particles, accounts for the momentum transfer between the gas and particles.

The flow Mach number is less than 0.01, thus we use a low-Mach approximation by neglecting the hydrodynamic pressure variation in comparison with the thermodynamic pressure. Therefore, the equation of state becomes

$$
P(t)=\rho_{a} R T \text {. }
$$

The outlet of the radiated domain is connected to the atmosphere and there is no heat transfer to the periodic domain. Therefore, in our calculation $P=$ $103,680 \mathrm{~Pa}$. Additionally, the energy equation for the gas phase is

$$
\frac{\partial}{\partial t}\left(\rho_{a} C_{\mathrm{v}} T\right)+\nabla \cdot\left(\rho_{a} \boldsymbol{u} C_{\mathrm{p}} T\right)=\nabla \cdot(k \nabla T)+\sum_{p} s_{p} \delta\left(\boldsymbol{x}-\boldsymbol{x}_{p}\right),
$$

in which $s_{\mathrm{p}}$ is given in the following and $k=k(T)$ is considered to be a function of temperature 22. In solving Equation 6, adiabatic and zero-Neumann BCs are imposed at the walls and outlet boundaries, respectively. 
The particle phase is formulated using a Lagrangian framework, in which

$$
\dot{\boldsymbol{x}}_{p}=\boldsymbol{u}_{p}
$$

and

$$
m_{p} \dot{\boldsymbol{u}}_{p}=\boldsymbol{g}-\boldsymbol{f}_{p}
$$

The smallest flow length scale at the center of the duct, estimated based on

the average dissipation, is $1.81 \times 10^{-4}$. This is 15 times larger than the diameter of particles. Also, the viscous length scale is $1.17 \times 10^{-4}$, which is 10 times larger than the diameter of particles. These larger flow structures in comparison with the particle size allows us to neglect the particle finite-size effects and use a point-particle model. Thus

$$
\boldsymbol{f}_{p}=m_{p} \tau_{p}^{-1}\left(\boldsymbol{u}_{p}-\boldsymbol{u}\right),
$$

which only accounts for the Stokes drag and neglects the history terms and nonlinear effects. These assumptions are justified by the high particle-to-air density ratio $\left(\rho_{\mathrm{p}} / \rho_{\mathrm{a}}=8900\right)$ as well as the small particle Reynolds number $\left(\rho_{\mathrm{a}}\left\|\boldsymbol{u}_{\mathrm{p}}-\boldsymbol{u}\right\| r_{\mathrm{p}} / \mu<1\right)$, respectively.

In Equation $9, \tau_{\mathrm{p}}=2 \rho_{\mathrm{p}} r_{\mathrm{p}}^{2} /(9 \mu)=3.81 \times 10^{-3} \mathrm{~s}$ is the particle momentum relaxation time. Normalizing this relaxation time by the bulk and wall time scale of flow produces $\mathrm{St}_{\eta}=1.82$ and $\mathrm{St}^{+}=4.35$, respectively. At this regime of $\mathrm{St}_{\eta}$, strong clustering of particles is expected at the center of the duct [23 25]. This phenomenon, which is also observed in this work, leads to depletion of particles from the vortical regions of flow and their accumulation in the high strain-rate regions [26. Also, relatively high value of $\mathrm{St}^{+}$is conducive to turbophoresis, which is the accumulation of particles near the walls $\left(y^{+}<10\right)$ [27, 28]. The presence of these high levels of particle concentration in the near wall region is also confirmed in this work. The particle clustering and turbophoresis introduces strong inhomogeneity in the particle concentration field and has direct implications in radiation transport modeling. 
The energy equation for the particle phase can be simplified due to their small thermal relaxation time. Hence, using the point-particle assumption

$$
m_{\mathrm{p}} C_{\mathrm{p}} \dot{T}_{\mathrm{p}}=\dot{Q}_{\mathrm{p}, \mathrm{OT}}-s_{\mathrm{p}},
$$

in which

$$
s_{\mathrm{p}}=2 \pi r_{\mathrm{p}} k \mathrm{Nu}\left(T_{\mathrm{p}}-T\right),
$$

and $\dot{Q}_{\mathrm{p}, \mathrm{OT}}=1.41 \times 10^{-4} \mathrm{~W}$ and zero in the radiated and periodic domains, respectively. This radiative heat transfer to each particle is calculated based on the low optical thickness assumption. This assumption is only employed in the DNS to obtain the particle distribution field in the radiated domain and is relaxed in the following sections. Analogous to Equation 9, Equation 11 is based on the assumption that the particle Reynolds number is small and flow around the particles can be well described by the Stokes equation.

A finite-volume formulation is used to solve Equations 2, 3, and 6, All governing equations are implemented in an in-house code that is second order accurate in space. A non-uniform $120 \times 80 \times 80$ rectilinear grid is generated for these calculations that is uniform in the streamwise direction and non-uniform in the wall-normal directions with the first grid point at $y^{+}=0.6$.

For the time integration, fourth order Runge-Kutta scheme is used. A fractional-step method is employed to impose conservation of mass [29. $\rho_{\mathrm{a}}$ and $\rho_{\mathrm{a}} \boldsymbol{u}$ are considered as the state variables.

Equation 6 is re-formulated as a constraint for the conservation of mass equation. Therefore, at each iteration of the Runge-Kutta scheme, a system of linear equations is solved to correct the velocity field with its right-hand-side depending on the energy equation. This linear system, which is non-symmetric due to the density variation and grid non-uniformity, is solved using a specialized geometric multigrid technique 30 .

The position, velocity, and temperature of the Lagrangian particles are integrated using the same time integration scheme as the gas phase. Particle-wall 
collisions are assumed to be elastic and the walls are assumed to be smooth. The gas and particle equations are fully coupled to ensure $4^{\text {th }}$ order accuracy in time.

To ensure statistical convergence, equations are integrated for approximately 100 flow-through-times. These computations are run in parallel using 96 processors, costing 14,000 CPU-hours.

\subsection{Monte Carlo Ray Tracing}

The MCRT code used to solve the RTE is based on previous work involving the simulation of radiative transfer in the plume of a solid rocket motor [8, 31. In the MCRT simulation, bundles of electromagnetic energy, referred to hereafter as rays, are emitted from the boundary surfaces and from the discrete particles. Emission from the boundary surfaces follows standard procedures described in [20, pp. 254-258]. Both standard sampling and stratified sampling in wavelength space are implemented in the code. The latter is useful in simulations where spectrally resolved radiative intensity is desired, rather than just the net radiative intensity. Since the heat flux to the particles is a function of the net radiative intensity, standard wavelength sampling is used in this work.

A user-specified number of rays is emitted from randomly selected particles in the domain. The emissive power of a particle is given by Equation 12

$$
P_{\mathrm{p}, \mathrm{i}}=8 \pi^{2} r_{\mathrm{p}}^{2} h c_{\mathrm{o}}^{2} \int_{\Delta \eta_{\mathrm{i}}} \frac{\epsilon\left(T_{\mathrm{p}}, \eta_{\mathrm{i}}\right) \eta^{3}}{e^{h c_{\mathrm{o}} \eta / k_{\mathrm{B}} T_{\mathrm{p}}}-1},
$$

which is the integral of the temperature and wavelength-dependent emissivity over the blackbody emissive power distribution, multiplied by the surface area of the particle. Values of $\mathrm{P}_{\mathrm{p}, \mathrm{i}}$ are calculated at the beginning of the simulation for each wavelength bin, and interpolation is performed to compute the value of $\mathrm{P}_{\mathrm{p}, \mathrm{i}}$ for a given particle temperature.

The initial power of each ray is calculated using Equation 13 [31,

$$
P_{\mathrm{r}}=\frac{N_{\eta} N_{\mathrm{p}}}{N_{\mathrm{r}}} P_{\mathrm{p}, \mathrm{i}}
$$


In Equation 13, the initial power of a given ray is calculated by scaling the emissive power of the particle in a specific wavelength bin by a correction factor, $N_{p} / N_{r}$, to account for the fact the number of bundles generated is not exactly equal to the number of particles in the domain. Additionally, the factor of $\mathrm{N}_{\eta}$ accounts for the fact that the power in a specific wavelength bin, $\mathrm{P}_{\mathrm{p}, \mathrm{i}}$, might be much less than the total emissive power of the particle. Equation 13 allows for statistical convergence to a correct result in the limit that the number of rays traced is much larger than both the number of particles, and the number of wavelength bins.

Rays are traced through the domain following the procedure given in [20, pp. 259-261]. Scattering is modeled using the cell-based scattering coefficient which is a function of the particle density in a given cell. The scattering coefficient in each cell is calculated as

$$
\sigma_{\mathrm{sca}}=\pi r_{\mathrm{p}}^{2} n_{\mathrm{p}} Q_{\mathrm{sca}},
$$

where it is assumed that the particle field in each cell is uniformly distributed. Once the cell-based scattering coefficient is determined, the distance to the scattering event in the current cell is computed. If this distance is smaller than the distance to the nearest cell edge, the ray is scattered following the procedures in [20, pp. 698-699]. Otherwise, the ray is advanced to the nearest intersection point of the direction vector of the ray and the faces of the cell.

Absorption by particles is modeled using the method of energy partitioning [20, p. 263 and references therein], where the absorption coefficient is given on a per cell basis by Equation 15 .

$$
\sigma_{\mathrm{abs}}=\pi r_{\mathrm{p}}^{2} n_{\mathrm{p}} Q_{\mathrm{abs}} .
$$

Once the ray has completely traversed the current cell, the direction-averaged 270 radiative energy flux in that cell is incremented by the amount given by Equation 
16 31,

$$
\Delta G_{\mathrm{i}}=\frac{P_{\mathrm{r}}}{\sigma_{\mathrm{abs}} V_{\mathrm{c}}}\left[1-\exp \left(-\sigma_{\mathrm{abs}} D_{\mathrm{t}}\right)\right] .
$$

Rays are traced through the domain until they are absorbed on a boundary or contain less than $1 \%$ of their original power. Simulations in which this cut-off value is varied are performed to confirm that the results are independent of the chosen value, for a reasonable range of values. When either of these two criteria are met, the ray is removed from the simulation.

After all rays have been traced, the heat transfer rate for an individual particle is calculated using Equation 17

$$
\dot{Q}_{\mathrm{p}}\left(\boldsymbol{x}_{\mathrm{p}}\right)=\pi r_{\mathrm{p}}^{2} Q_{\mathrm{abs}} G\left(\boldsymbol{x}_{\mathrm{p}}\right) .
$$

In Equation 17, it is assumed that the efficiencies are independent of the direction of incident radiation so that Kirchoff's law is valid.

The code is verified using six different test cases that are summarized in 32. The test cases involve one-dimensional surfaces bounding free space 20 , pp. 179181], one-dimensional surfaces bounding absorbing, isotropically and anisotropically scattering media [33 35] 20, pp. 179-181], and a three-dimensional cube containing grey, inhomogeneous media [36, 37]. The solutions used for verification are either analytical solutions or results from benchmark cases. In order to verify the portions of the code involving discrete particles, these cases are simulated using a uniformly distributed, discrete particle medium. In all cases, the relative difference between the exact or benchmark solutions and the result produced by the MCRT code is less than $3 \%$.

The code is developed to run on memory-distributed parallel computer architectures using the KULL domain-decomposition method [38].

\subsection{Boundary Conditions}

The lamp source is modeled by treating the $\mathrm{Z}=0$ surface of the domain as a blackbody emitter with a temperature of $2500 \mathrm{~K}$. The lamp emission is assumed 
to be perfectly collimated. All other surfaces of the domain are treated as cold, black walls for the purposes of this initial study.

\section{Results}

All simulations are conducted in three dimensions. Calculations are initially

Figure 5 shows the transmission through the depth of the channel, normal to the direction of the collimated lamp radiation. The difference between the 

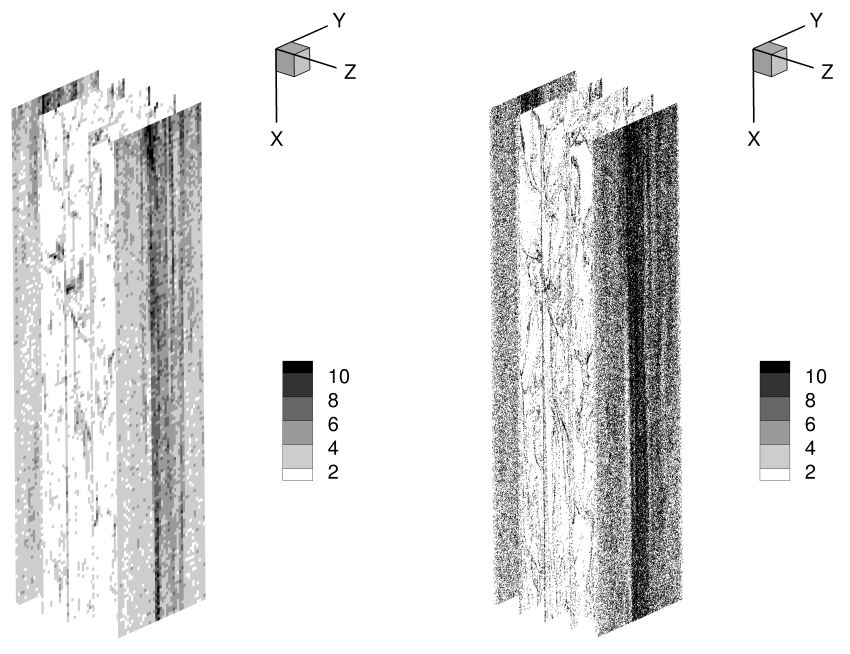
(a) $\Delta x=1 \mathrm{~mm}$
(b) $\Delta x=0.25 \mathrm{~mm}$

Figure 4: Particle number density $\left(n_{\mathrm{p}}, \mathrm{m}^{-3}\right)$ on planes normal to the direction of incident radiation for two different mesh sizes.

predicted transmission on each of the $0.5 \mathrm{~mm}, 0.25 \mathrm{~mm}$ and $0.125 \mathrm{~mm}$ meshes is within the $95 \%$ confidence interval associated with the mean transmission value, indicating that a globally grid independent solution has been obtained. Based on these results, the $0.5 \mathrm{~mm}$ mesh is used for the remainder of this study. This mesh has just over two million uniformly sized cells.

\subsection{Computational Requirements}

330

All simulations are carried out on the Flux high performance computing cluster at the University of Michigan. The required computational time for each simulation carried out using the $\Delta x=0.5 \mathrm{~mm}$ mesh is approximately 133 CPU hours on $242.60 \mathrm{GHz}$ Intel Xeon E5-2670 processors each with 4 GB of RAM and connected with 40 Gbps InfiniBand networking. 


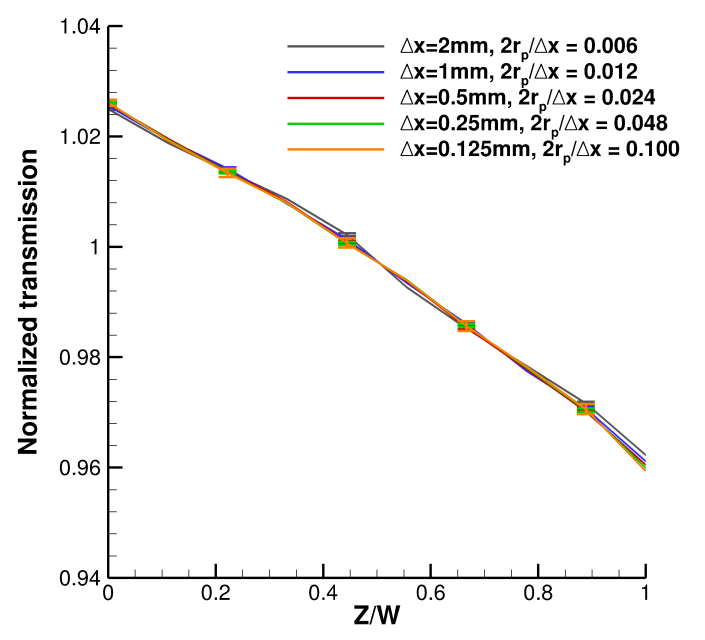

Figure 5: Total, normalized, transmitted radiation through the depth of the domain. incident radiation. Figure 7 shows the particle heat transfer rate on planes located at $\mathrm{Y} / \mathrm{W}=0.00625$ (near the channel wall) and $\mathrm{Y} / \mathrm{W}=0.5$ (center of the the channel). As expected, as the distance from the source increases, the particle heat transfer rate decreases. Areas on this figure associated with the 350

\subsection{Baseline Results}

Figure 6 shows contours of radiation intensity on planes normal to the incident radiation. The contours are normalized by the intensity of the lamp source. The intensity of the incident radiation is greater than that of the lamp near the source, due to backscattering of radiation from particles. As the distance from the source increases, the radiation intensity decreases. However, due to scattering, it does not decrease monotonically.

The heat transfer rate to each particle is a linear function of the radiation intensity, and is given by Equation 17. In Equation 17, $G\left(\boldsymbol{x}_{\mathrm{p}}\right)$ is the directionaveraged incident radiation, and is a function of position. Thus, the spatial variation in the predicted particle heat transfer rate is the same as that of the lowest contour value $\left(6 \times 10^{-5} \mathrm{~W}\right)$ are regions that do not contain any particles.

Figure 7 shows that there is more variation in the particle heat transfer rate 


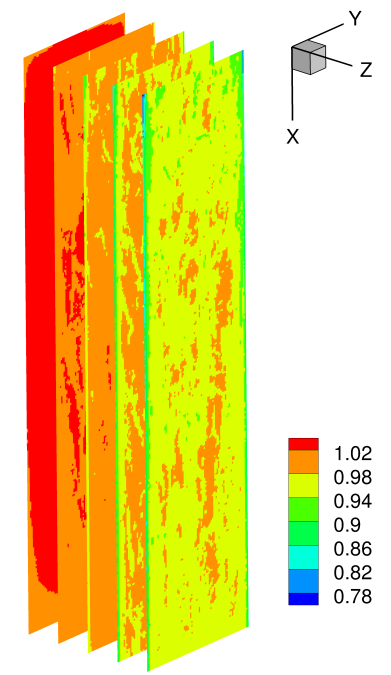

Figure 6: Normalized and directionally averaged radiation intensity $\left(G\left(\boldsymbol{x}_{\mathrm{p}}\right) / \mathrm{I}_{1}\right)$ on planes normal to the incident radiation.

on the $\mathrm{Y} / \mathrm{W}=0.00625$ plane, and fewer regions that are void of particles. This is because turbophoresis causes the particle density near the walls of the domain to be higher than in the center of the domain. This is illustrated in Figure 8 , which shows contours of particle number density on the $\mathrm{Y} / \mathrm{W}=0.00625$ and $\mathrm{Y} / \mathrm{W}=0.5$ planes. Again, areas on this figure associated with the lowest contour value $\left(0.01 \times 10^{10} \mathrm{~m}^{-3}\right)$ are regions that do not contain any particles.

In the optically thin approximation, absorption and scattering of radiation is neglected, and the heat transfer rate for each particle is given by

$$
\dot{Q}_{\mathrm{p}, \mathrm{OT}}=\pi r_{\mathrm{p}}^{2} Q_{\mathrm{abs}} I_{1}
$$

where $I_{1}$ is the radiation intensity coming from the lamp source and is independent of the positions of the particles. The error in the particle heat transfer rate throughout the domain due to the use of the optically thin approximation, 


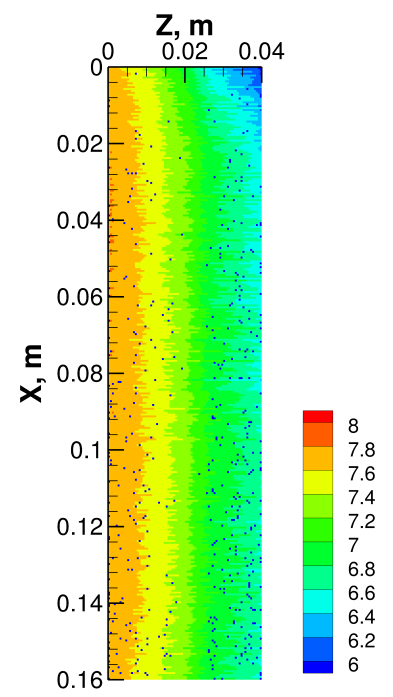

(a) $\mathrm{Y} / \mathrm{W}=0.00625$

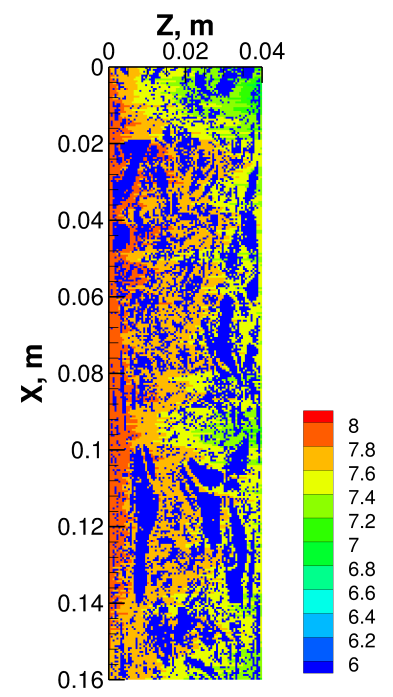

(b) $\mathrm{Y} / \mathrm{W}=0.5$

Figure 7: Particle heat transfer rate $\left(\dot{Q}_{\mathrm{p}} \times 10^{-5}, \mathrm{~W}\right)$ on two different extraction planes.

$\varepsilon_{\mathrm{OT}}$, is given by Equation 19

$$
\varepsilon_{\mathrm{OT}}=\frac{G_{\mathrm{p}}\left(\boldsymbol{x}_{\mathrm{p}}\right)-I_{1}}{I_{\mathrm{l}}},
$$

where the incident radiation is used to quantify the error in the particle heat

transfer rate. This choice does not affect the result, since the terms $\pi r_{\mathrm{p}}^{2} Q_{\mathrm{abs}}$ appear in both Equations 17 and 18. If the computed heat transfer rate had been used in Equation 19, the error would reach unity in areas of the domain that do not contain particles, making visualization of the error in regions that do contain particles (and the only regions where this error has meaning) more difficult.

Figure 9 shows contours of $\varepsilon_{\mathrm{OT}}$ on both the near-wall slice and the slice in the center of the channel. The optically thin approach results in an underestimate of the particle heat transfer rate near the lamp source, because backscattering of radiation towards the source is neglected. The optically thin approach results 


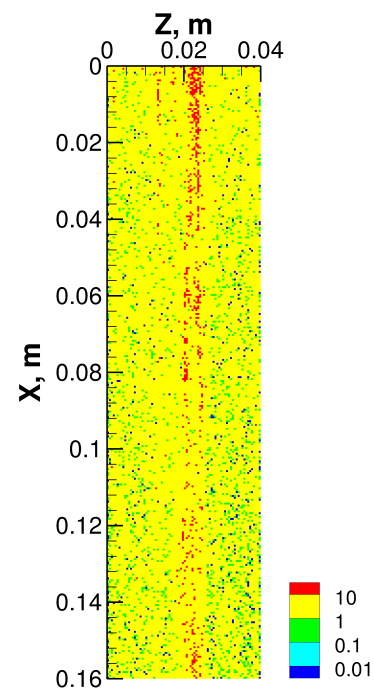

(a) $\mathrm{Y} / \mathrm{W}=0.00625$

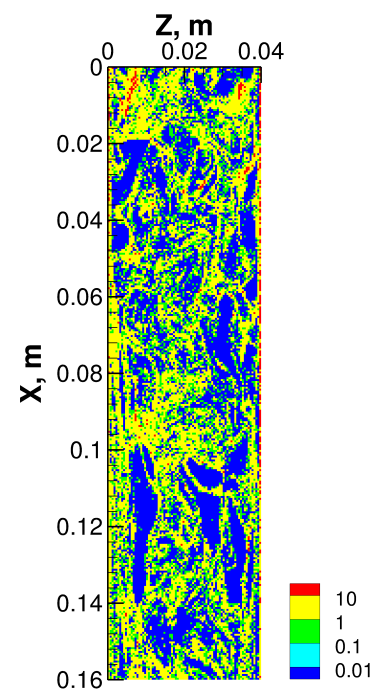

(b) $\mathrm{Y} / \mathrm{W}=0.5$

Figure 8: Particle number density $\left(\mathrm{n}_{\mathrm{p}} \times 10^{10}, \mathrm{~m}^{-3}\right)$ on two different extraction planes.

375 domain, at $\mathrm{Z} / \mathrm{D}=1$, because extinction of radiation as it traverses the domain is neglected. The errors in the entire domain range from $\varepsilon_{\mathrm{OT}}=-0.35$ to $\varepsilon_{\mathrm{OT}}$ $=0.035$. The regions of maximum error are located near the side walls and the back wall of the domain, where the largest particle densities are found and therefore the radiation path length is shortest. These substantial differences in the predicted particle heat transfer rate may affect the numerical predictions of turbulent, particle-laden flow, and therefore warrant the use of a more detailed radiative transfer model in future analysis of these types of flows.

\subsection{Assessment of the Geometric Optics Assumption}

385

To assess the validity of the geometric optics assumption used to construct the optical model, optical properties are calculated using the Mie-scattering code developed by Modest [20, pp. 390-393] and [39. A comparison of the extinction 


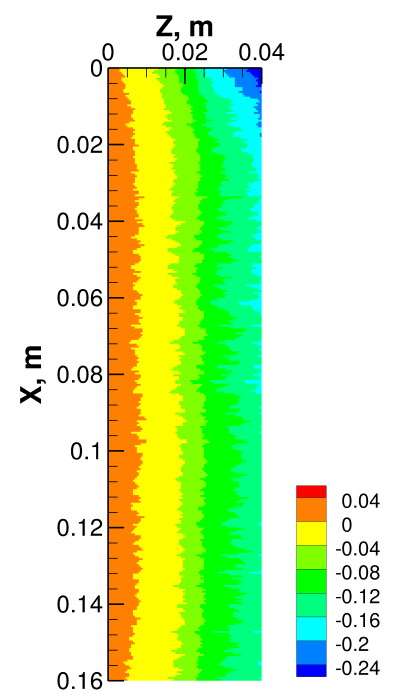

(a) $\mathrm{Y} / \mathrm{W}=0.00625$

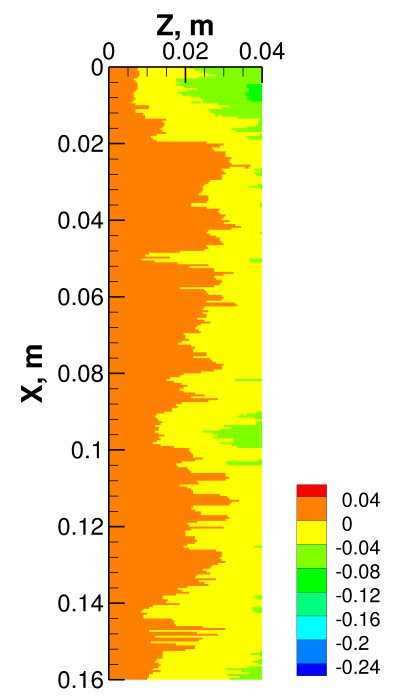

(b) $\mathrm{Y} / \mathrm{W}=0.5$

Figure 9: Error in predicted particle heat transfer rate $\left(\varepsilon_{\mathrm{OT}}\right)$ on two different extraction planes.

coefficients predicted using Mie theory and from the geometric optics model is shown in Figure 10 for the size parameter range of interest in this study. The scattering efficiency predicted using geometric optics is lower than that predicted using Mie theory because forward scattering is treated as transmission in the geometric optics approach, and accounts for one half of the total extinction efficiency. The two approaches give similar values at small wavelengths (large size parameter), but differ by as much as $30 \%$ at larger wavelengths (small size parameter). The peak of the blackbody spectrum for the collimated lamp source is located at approximately $1 \mu \mathrm{m}$, corresponding to a size parameter of 40 . This size parameter is in the region of disagreement between the optical properties calculated using geometric optics and Mie theory.

Figure 11 shows the phase function for $\mathrm{r}_{\mathrm{p}}=6 \mu \mathrm{m}$ nickel particles at a wavelength of $1 \mu \mathrm{m}$ predicted using Mie theory. The isotropic phase function 


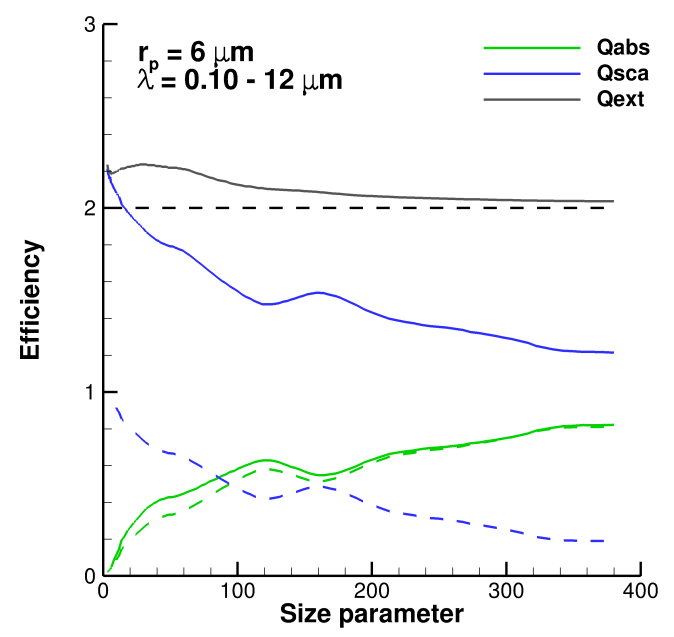

Figure 10: Comparison of optical efficiencies predicted using geometric optics and Mie theory. Solid line - Mie theory; dashed line - geometric optics.

is included on this figure for comparison purposes. The phase function is very strongly forward peaked, as is typical for relatively large metallic particles. This phase function can be modeled using the Delta-Eddington approximate phase function [16], which involves separating the forward scattering peak and treating it as transmission.

In order to investigate the error associated with the use of the geometric optics assumption, two additional simulations are conducted at a wavelength of $1 \mu \mathrm{m}$. In the first, the geometric optics assumption is used and the particle emissivity (and hence, absorption efficiency) is set to 0.303 . The reflectivity (and hence, scattering efficiency) is $1-\epsilon=0.697$, and an isotropic phase function is used. In the second simulation, results from the Mie theory analysis are used to set the absorption efficiency to 0.396 and the scattering efficiency to 0.745 . The Delta-Eddington approximation is used to separate the forward scattering peak from the rest of the phase function and model it as transmission. Thus, an 415 isotropic phase function is used in the second simulation as well. These input parameters are summarized in Table 3 , where $Q_{\text {sca,DE }}$ indicates the scattering 


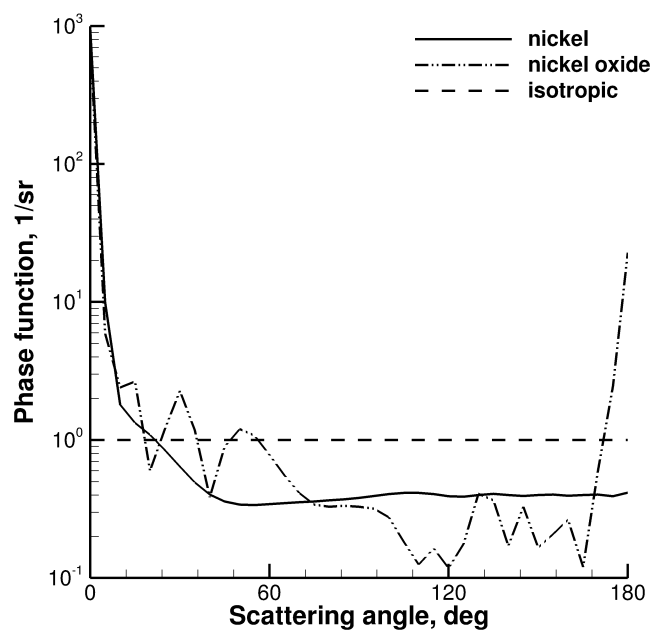

Figure 11: Phase functions for nickel, nickel oxide, and isotropically scattering particles.

Table 3: Scattering and absorption efficiencies from geometric optics and Mie theory for $\lambda=$ $1 \mu \mathrm{m}$.

\begin{tabular}{lrrr}
\hline \hline & $\mathrm{Q}_{\mathrm{abs}}$ & $\mathrm{Q}_{\text {sca }}$ & $\mathrm{Q}_{\text {sca,DE }}$ \\
\hline Geometric optics & 0.303 & 0.697 & 0.697 \\
Mie theory & 0.396 & 1.84 & 0.745 \\
\hline \hline
\end{tabular}

efficiency used in simulations where the phase function is modeled using the Delta-Eddington approximation.

The minimum and maximum values of particle heat transfer rate in the computational domain obtained using the geometric optics assumption and Mie theory are shown in Table 4 Particles absorb as much as $30 \%$ more radiation at $\lambda=1 \mu \mathrm{m}$ in the Mie theory approach, since this quantity is directly proportional to the absorption efficiency. Thus, if less than $30 \%$ error in the particle heat transfer rate is required, Mie theory should be used to determine the optical properties of the dispersed nickel particle phase. 
Table 4: Particle heat transfer rates predicted from three simulations with $\lambda=1 \mu \mathrm{m}$.

\begin{tabular}{lrrr}
\hline \hline & $\min . \dot{Q}_{\mathrm{p}}, \mathrm{W}$ & $\max . \dot{Q}_{\mathrm{p}}, \mathrm{W}$ & $\max . \delta_{\dot{Q}_{\mathrm{p}}}$ relative to GO \\
\hline Geometric optics & $9.21 \times 10^{-5}$ & $1.10 \times 10^{-4}$ & - \\
Mie theory & $1.17 \times 10^{-4}$ & $1.43 \times 10^{-4}$ & $30 \%$ \\
Oxidized particle & $1.55 \times 10^{-4}$ & $1.95 \times 10^{-4}$ & $77 \%$ \\
\hline \hline
\end{tabular}

\subsection{Effects of Particle Oxidation}

Given that the particles are nickel and are subject to a high temperature environment, it is possible that an oxide layer will form on the surface of the particles. Mie theory for a coated sphere is used to address the potential effects of surface oxidation on the optical properties of the particles 40, pp. 181183]. Since it is unlikely that the complex index of refraction for the $\mathrm{NiO}$ layer that may be formed will be known exactly, an approximate complex index of refraction, $m=2.0+1 \times 10^{-6} \mathrm{i}$, is used in this analysis [41].

Figures 12 and 13 show the extinction and absorption efficiencies for $r_{\mathrm{p}}=6 \mu \mathrm{m}$ nickel particles coated with various thicknesses of a dielectric material. Lines labeled "oxidized particle" are results for a partially oxidized nickel particle, where the radius of the nickel portion of the particle is $r_{\mathrm{p}}=6 \mu \mathrm{m}$. For example, in Figure 12, a pure nickel particle is represented by the black line, a completely oxidized particle is represented by the blue line, and a partially oxidized particle 440 is represented by the red line. All results are generated at a wavelength of $1 \mu \mathrm{m}$. The results for $r_{\mathrm{p}}=6 \mu \mathrm{m}$ correspond to an unoxidized particle with a radius of $6 \mu \mathrm{m}$, so the figures show the behavior of the optical efficiencies as a function of the thickness of the oxide layer relative to particles of uniform composition and with the same radius.

${ }_{445}$ There is experimental data for thin nickel films that suggests that a $4 \mu \mathrm{m}$ thick oxide layer forms after two hours of exposure in an air furnace at $900^{\circ} \mathrm{C}$ [42. For this reason, $4 \mu \mathrm{m}$ is the maximum layer thickness considered in the analysis. However, for clarity, the abscissa of Figures 12 and 13 are truncated at 
a particle radius of $8 \mu \mathrm{m}$. At the size parameters considered, there is a primary oscillation in the extinction efficiency of the dielectric onto which secondary oscillations are superimposed. This is the cause of the maxima and minima in the results that are presented.

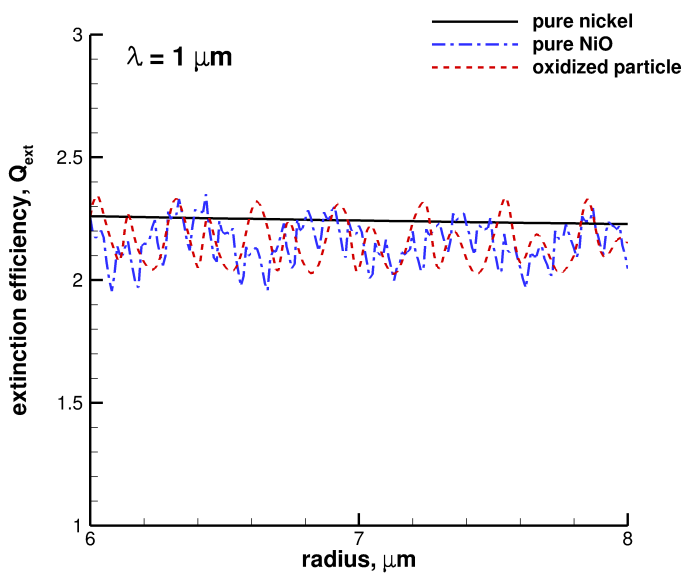

Figure 12: Extinction efficiency of $r_{\mathrm{p}}=6 \mu \mathrm{m}$ particles as a function of total particle radius.

Figure 11 shows the phase function for both the pure nickel and completely oxidized particles. The phase function of the $\mathrm{NiO}$ particle contains both a forward and backward scattering peak, which is expected to be present in the phase function of a partially oxidized particle as well. The asymmetry parameter, $g$, can be used in a simplified phase function such as Henyey-Greenstein or DeltaEddington to capture the forward scattering peak. However, these approximate phase functions will not capture the backscattering peak in the dielectric particle response that is shown in Figure 11.

The transit time of particles through the irradiated section of the geometry is expected to be less than one second, so if an oxide layer forms it will likely be during storage at room temperature. The results of the experiment in 42 indicate that the thickness of this oxide layer is likely to be very small. Assuming that oxide does form with a thickness of $1 \%$ of the particle radius, it will have a thickness of $t_{\mathrm{ox}}=0.06 \mu \mathrm{m}$. Table 5 shows the scattering and absorption 


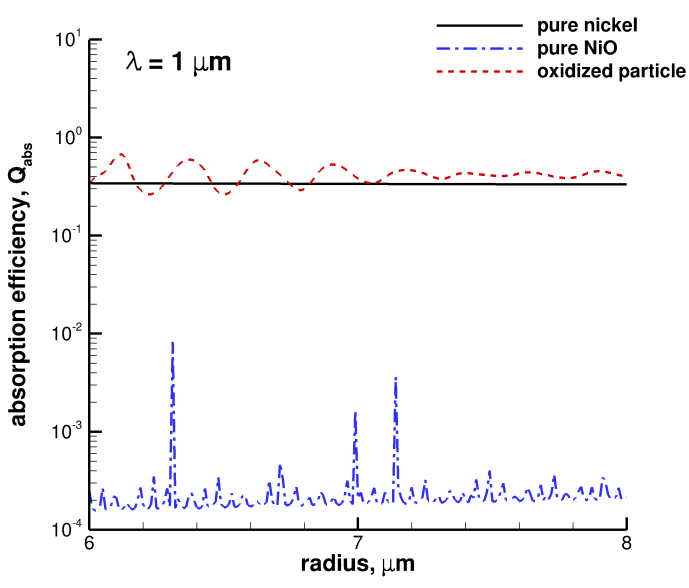

Figure 13: Absorption efficiency for $r_{\mathrm{p}}=6 \mu \mathrm{m}$ particles as a function of total particle radius.

efficiencies, and the asymmetry parameter $\mathrm{g}$, for the oxidized particle and the unoxidized particle at $\lambda=1 \mu \mathrm{m}$. Table 5 shows that the $t_{\mathrm{ox}}=0.06 \mu \mathrm{m}$ thick oxide layer increases the absorption efficiency by $34 \%$ at $1 \mu \mathrm{m}$, but decreases the scattering efficiency by only $7 \%$. The total extinction efficiency is unchanged. The phase function for the oxidized particle is more strongly forward peaked than that of the pure nickel particle, as indicated by the increase in the asymmetry parameter.

In order to assess the effect of the oxide layer on the particle heat transfer rate, an additional simulation is performed using the optical properties in Table 5 for the oxidized particle. Again, the Delta-Eddington approximation is used to separate the forward scattering peak from the rest of the phase function and model it as transmission. As demonstrated in Section 4.4 the change in the heat transfer rate due to oxidation is expected to be of a similar magnitude as the change in the absorption coefficient. The increased number of forward scattering events is expected to slightly mitigate the effect of the increased absorption efficiency, as the path length of a ray from the lamp that undergoes successive forward scattering events is, in general, shorter than that of a ray that undergoes scattering isotropically. Table 4 shows that the maximum predicted difference 
Table 5: Mie efficiencies and asymmetry parameter for an unoxidized particle and an oxidized particle with $t_{\mathrm{ox}}=0.06 \mu \mathrm{m}$.

\begin{tabular}{lrrrr}
\hline \hline & $Q_{\text {abs }}$ & $Q_{\text {sca }}$ & $g$ & $Q_{\text {sca }, \mathrm{DE}}$ \\
\hline unoxidized & 0.396 & 1.84 & 0.606 & 0.745 \\
$t_{o x}=0.06 \mu \mathrm{m}$ & 0.532 & 1.70 & 0.693 & 0.522 \\
\hline \hline
\end{tabular}

485 with the geometric optics assumption is $77 \%$. This is clearly a significant result, but it is directly dependent on the properties of the oxide layer.

Figure 13 shows that the absorption efficiency of the oxide particle varies from a value of $Q_{\mathrm{abs}}=0.3$ to $Q_{\mathrm{abs}}=0.8$ over a thickness range of $t_{\mathrm{ox}}=0 \mu \mathrm{m}$ to $t_{\mathrm{ox}}=2 \mu \mathrm{m}$. This is a $-25 \%$ to $100 \%$ variation relative to the unoxidized result. Since the particle heat transfer rate is proportional to the absorption efficiency, the variation of the predicted heat transfer rate due to uncertainty in the thickness of the oxide layer is expected to be of a similar magnitude.

\subsection{Effects of Particle Shape}

495

The assumption of a perfectly spherical particle is an idealization, and deviations from sphericity may change the optical properties of the particles. To provide a first assessment of the magnitude of this effect, the T-matrix approach [43, 44] is used to numerically solve the Maxwell equations in order to compute light scattering by non-spherical, randomly oriented particles. The non-spherical particles are formed by deforming a sphere by means of a Chebyshev polynomial of degree $n$ [45]. The shape of the two-dimensional cross section of the deformed particles is given in a polar-coordinate system by

$$
r(\theta)=r_{o}\left[1+a T_{n}(\cos \theta)\right]
$$

The deformation parameter, $a$, can be negative or positive but must have a magnitude that is less than one. 

"rough" particles, where the size of the roughness and the frequency of the peaks and valleys is controlled by the deformation parameter and the degree of the Chebyshev polynomial. In this work, particles are studied with orders ranging from two to twenty. Images of these particles are given in Figure 14.

Table 6] shows the optical properties for each particle shape, including equal volume spheres with $r_{\mathrm{p}}=6 \mu \mathrm{m}$. In cases where the shape of the particle with $(n,+a)$ is not visibly different than that with $(n,-a)$, only the results for the particle with $(n,+a)$ are reported. In all cases, the relative differences between the properties predicted by Mie theory and those predicted using the T-matrix approach are less than $4 \%$. The effect on the predicted heat flux to the particles will be of similar magnitude, and therefore much less significant than the effect of surface oxidation. The absorption and scattering efficiencies for the non-spherical particles are larger than those for the spherical particles in all cases. The phase function asymmetry parameter is smaller than that of the spherical particles in all cases. These trends are consistent with an earlier study that showed that nonsphericity tends to increase absorption and extinction efficiencies over a range of size parameters and particle shapes, while reducing the degree of asymmetry of the scattering phase function [45].

\section{Conclusions}

Three-dimensional Monte Carlo ray tracing simulations are applied to model radiative transport through a channel containing preferentially-concentrated, micronscale nickel particles interacting with a non-participating turbulent flow of air. The flow of the turbulent air and particles is considered in a separate computational model, using Direct Numerical Simulation of the Navier-Stokes equations coupled to Lagrangian tracking of point-particles. The predicted radiative heat transfer rate to the particles is studied and compared to that predicted using the optically thin approximation. One outcome of this study is a verified MCRT code for radiative transfer in a dispersed particle phase that can be used to pro- 


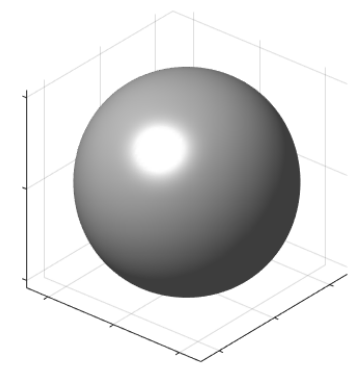

(a) $(n, a)=(2,0.1)$

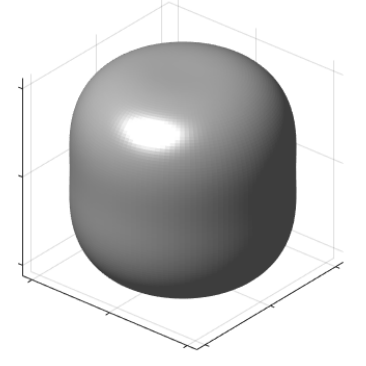

(c) $(n, a)=(4,-0.1)$

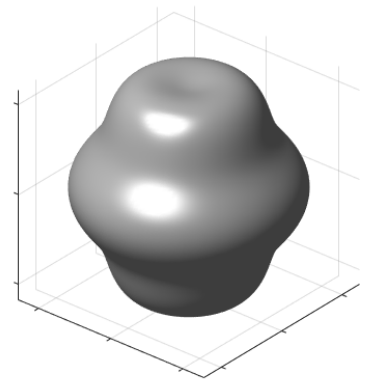

(e) $(n, a)=(6,-0.1)$

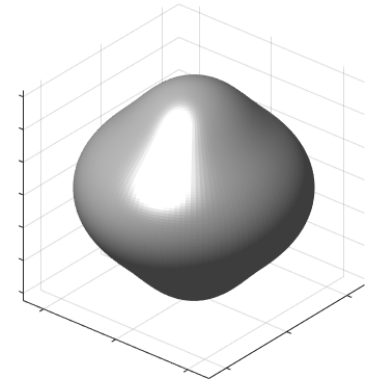

(b) $(n, a)=(4,0.1)$

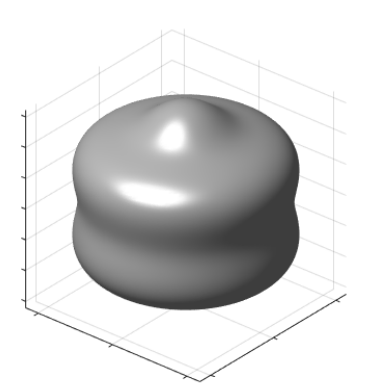

(d) $(n, a)=(6,0.1)$

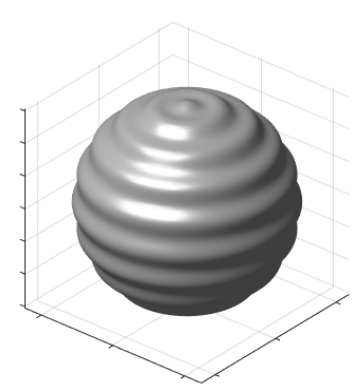

(f) $(n, a)=(20,0.02)$

Figure 14: Chebyshev particles with $T_{n}(a)$. 
Table 6: Optical properties for Chebyshev particles and equal volume spheres.

\begin{tabular}{lrrrrrr}
\hline \hline particle shape & $Q_{\text {abs }}$ & $Q_{\text {sca }}$ & $g$ & $\delta_{\dot{Q}_{\text {abs }}}$ & $\delta_{\dot{Q}_{\text {sca }}}$ & $\delta_{g}$ \\
\hline sphere & 0.396 & 1.84 & 0.606 & - & - & - \\
$(n, a)=(2,0.1)$ & 0.398 & 1.84 & 0.606 & 0.418 & 0.434 & -0.0157 \\
$(n, a)=(4,0.1)$ & 0.402 & 1.86 & 0.605 & 1.37 & 1.32 & -0.224 \\
$(n, a)=(4,-0.1)$ & 0.402 & 1.86 & 0.605 & 1.32 & 1.28 & -0.227 \\
$(n, a)=(6,0.1)$ & 0.407 & 1.88 & 0.601 & 2.85 & 2.37 & -0.889 \\
$(n, a)=(6,-0.1)$ & 0.407 & 1.88 & 0.601 & 2.79 & 2.21 & -0.895 \\
$(n, a)=(20,0.02)$ & 0.409 & 1.84 & 0.600 & 3.25 & 0.464 & -0.997 \\
\hline \hline
\end{tabular}

vide benchmark solutions, supporting the development of computer codes using

approximate solution techniques such as DOM.

Turbophoresis causes the particle density near the walls of the domain to be higher than in the center of the domain. This leads to the incoming radiation being absorbed non-uniformly throughout the channel, with more of the incoming radiation being absorbed in the near-wall regions of the channel than in the center of the channel. Results of the MCRT simulations show that the optically thin approximation used in previous work results in errors of up to $35 \%$ in the particle heat transfer rate for the particle-laden flow environment investigated in this study. The optically thin approach results in an underestimate of the particle heat transfer rate near the radiation source, and an overestimate of the particle heat transfer rate near the back wall of the domain. The regions of maximum error are located near the side walls and the back wall of the domain, where the largest particle densities are found and therefore the radiation path length is shortest. Substantial differences in particle heat transfer rate may affect the results of numerical predictions of turbulent, multiphase flow. Thus, this result motivates the use of more detailed radiative transfer models in future computational studies of these types of flows.

Sensitivity of the results to some of the assumptions made during the con- 
struction of the physical models is investigated. For the conditions considered in this work, the use of the geometric optics assumption to construct the optical model for nickel particles results in a 30\% error in the predicted particle heat transfer rate relative to the case where Mie theory is used. Additionally, the formation of an oxide layer with a thickness of $1 \%$ of the particle radius is shown to increase the absorption efficiency of the particles by $34 \%$. Lastly, the effect of non-sphericity of the particles is assessed using the T-matrix method and Chebyshev particles of various shapes in order to simulate surface deformation and roughness. It is found that changes in the optical properties due to roughness are less than $4 \%$ for the particle shapes considered in this study.

The results of this study motivate a more detailed investigation of the uncertainty in radiative heat transfer predictions due to particle oxidation and particle shape. Additional sources of uncertainties, such as particle size distributions, and uncertainties in refractive index should also be included in future uncertainty quantification efforts.

\section{Acknowledgements}

This material is based upon work supported by the Department of Energy National Nuclear Security Administration under Award Number DE-NA00023731. E. Farbar would like to acknowledge Ms. Nomita Vazirani for her help generating cross sections, Professor Mark Flanner for sharing his code for calculating coated sphere properties, Mr. Ari Frankel for useful discussions regarding radiation transport in discrete media, Dr. Jonathan Burt for useful discussions 575 regarding radiation modeling in flows containing solid particles, and Mr. Andrew Banko for answering questions regarding experimental design.

\section{References}

[1] F. J. Miller, R. W. Koenigsdorff, Thermal modeling of a small-particle solar central receiver, Journal of Solar Energy Engineering 122 (2000) 23-29. 
[7] S. K. Hiroaki Watanabe, Ryoichi Kurose, H. Pitsch, Effects of radiation on spray flame characteristics and soot formation, Combustion and Flame 152 (2008) 2-13.

[8] J. M. Burt, I. D. Boyd, High altitude plume simulations for a solid propel-

[9] R. Duval, A. Soufiani, J. Taine, Coupled radiation and turbulent multiphase flow in an aluminised solid propellant rocket engine, Journal of Quantitative Spectroscopy and Radiative Transfer 84 (2004) 513-526.

[10] K. Matsuda, R. Onishi, R. Kurose, S. Komori, Turbulence effect on cloud 605 of a prototype solid particle receiver: On-sun testing and model validation, Journal of Solar Energy Engineering 132 (2), 021008.

[3] H. H. Klein, J. Karni, R. Ben-Zvi, R. Bertocchi, Heat transfer in a directly irradiated solar receiver/reactor for solid-gas reactions, Solar Energy 81 (2007) 1227-1239.

[4] H. Chen, Y. Chen, H.-T. Hsieh, N. Siegel, Computational Fluid Dynamics modeling of gas-particle flow within a solid-particle solar receiver, Journal of Solar Engineering 129 (2007) 160-170.

[5] W. Lipiński, A. Z'Graggen, A. Steinfeld, Transient radiation heat transfer within a nongray nonisothermal absorbing-emitting-scattering suspension of reacting particles undergoing shrinkage, Numerical Heat Transfer, Part B 47 (2005) 443-457.

[6] W. Lipiński, D. Thommen, A. Steinfeld, Unsteady radiative heat transfer within a suspension of $\mathrm{ZnO}$ particles undergoing thermal dissociation, Chemical Engineering Science 61 (2006) 7029-7035.

lant rocket, AIAA Journal 45 (12) (2007) 2872-2884.

radiation, Physical Review Letters 108, 224502. 
[11] R. A. Shaw, Particle-turbulence interactions in atmospheric clouds, Annual Review of Fluid Mechanics 35 (2003) 183-227.

[12] A. Collin, P. Boulet, D. Lacroix, G. Jeandel, On radiative transfer in water spray curtains using the discrete ordinates method, Journal of Quantitative Spectroscopy and Radiative Transfer 82 (2005) 85-110.

[13] R. Zamansky, F. Coletti, M. Massot, A. Mani, Radiation induces turbulence in particle-laden fluids, Physics of Fluids 26, 071701.

[14] D. Baillis, J.-F. Sacadura, Thermal radiation properties of dispersed media: theoretical prediction and experimental characterization, Journal of Quantitative Spectroscopy and Radiative Transfer 67 (2000) 327-363.

[15] J. T. Farmer, J. R. Howell, Comparison of Monte Carlo strategies for radiative transfer in participating media, Advances in Heat Transfer 31 (1998) 333-429.

[16] J. T. Farmer, J. R. Howell, Monte Carlo prediction of radiative heat transfer in inhomogeneous, anisotropic, nongray media, Journal of Thermophysics and Heat Transfer 8 (1) (1994) 133-139.

[17] Y. Shuai, S. K. Dong, H. P. Tan, Simulation of the infrared radiation characteristics of high-temperature exhaust plume including particles using the backward Monte Carlo method, Journal of Quantitative Spectroscopy and Radiative Transfer 95 (2005) 231-240.

[18] S. Balachandar, J. K. Eaton, Turbulent dispersed multiphase flow, Annual Review of Fluid Mechanics 42 (2010) 111-133.

[19] E. D. Palik (Ed.), Handbook of Optical Constants of Solids, Academic Press, 1985.

${ }_{630}[20]$ M. F. Modest, Radiative Heat Transfer, 3rd Edition, Elsevier, 2013. 
[21] W. Sutherland, LII. the viscosity of gases and molecular force, The London, Edinburgh, and Dublin Philosophical Magazine and Journal of Science 36 (223) (1893) 507-531.

[22] R. C. Weast, Handbook of chemistry and physics, The American Journal of the Medical Sciences 257 (6) (1969) 423.

[23] J. R. Fessler, J. D. Kulick, J. K. Eaton, Preferential concentration of heavy particles in a turbulent channel flow, Physics of Fluids 6 (11) (1994) 37423749.

[24] J. K. Eaton, J. Fessler, Preferential concentration of particles by turbulence, International Journal of Multiphase Flow 20 (1994) 169-209.

[25] A. Aliseda, A. Cartellier, F. Hainaux, J. C. Lasheras, Effect of preferential concentration on the settling velocity of heavy particles in homogeneous isotropic turbulence, Journal of Fluid Mechanics 468 (2002) 77-105.

[26] S. L. Rani, S. Balachandar, Evaluation of the equilibrium Eulerian approach for the evolution of particle concentration in isotropic turbulence, International Journal of Multiphase Flow 29 (12) (2003) 1793-1816.

[27] D. Kaftori, G. Hetsroni, S. Banerjee, Particle behavior in the turbulent boundary layer. II. velocity and distribution profiles, Physics of Fluids 7 (5) (1995) 1107-1121.

[28] P. Papavergos, A. Hedley, Particle deposition behaviour from turbulent flows, Chemical Engineering Research \& Design 62 (5) (1984) 275-295.

[29] J. Kim, P. Moin, Application of a fractional-step method to incompressible Navier-Stokes equations, Journal of Computational Physics 59 (2) (1985) 308-323.

${ }_{655}$ [30] M. Esmaily-Moghadam, L. Jofre, G. Iaccarino, A. Mani, A scalable geometric multigrid method for non-symmetric linear systems arising from elliptic equations, Annual Research Brief (2015) 211-223. 
[31] J. M. Burt, I. D. Boyd, A Monte Carlo radiation model for simulating rarefied multiphase plume flows, in: 38th AIAA Thermophysics Conference, AIAA, 2005, paper 2005-4691.

[32] X. Sun, Reverse Monte Carlo ray tracing for radiative heat transfer in combustion systems, Ph.D. thesis, University of Utah (2009).

[33] I. W. Busbridge, S. E. Orchard, Reflection and transmission of light by a thick atmosphere according to a phase function: $1+\mathrm{x} \cos \theta$, The Astrophysical Journal 149 (1967) 655-664.

[34] R.Siegel, Transient radiative cooling of a droplet-filled layer, ASME Journal of Heat Transfer 109 (1987) 159-164.

[35] M. A. Heaslet, R. F. Warming, Radiative transport and wall temperature slip in an absorbing planar medium, International Journal of Heat and Mass Transfer 8 (1965) 979-994.

[36] S. P. Burns, M. A. Christen, Spatial domain-based parallelism in large-scale participating-media, radiative transport applications, Numerical Journal of Heat Transfer, Part B 31 (1997) 401-421.

[37] P.-F. Hsu, J. T. Farmer, Benchmark solutions of radiative heat transfer within nonhomogeneous participating media using the Monte Carlo and YIX method, ASME Journal of Heat Transfer 119 (1997) 185-188.

[38] T. A. Brunner, T. J. Urbatsch, T. M. Evans, N. A. Gentile, Comparison of four parallel algorithms for domain decomposed implicit Monte Carlo, Journal of Computational Physics 212 (2006) 527-539.

680 [39] M. F. Modest, mmmie.f [cited April 4, 2016]. URL http://booksite.elsevier.com/9780123869449

[40] C. F. Bohren, D. R. Huffman, Absorption and Scattering of Light by Small Particles, Wiley-VCH, 2004. 
[41] D. W. Lynch, R. Rosei, J. H. Weaver, Infrared and visible optical properties of single crystal Ni at 4K, Solid State Communications 9 (1971) 2195-2199.

[42] T. R. Fu, P. Tan, J. Ren, H. S. Wang, Total hemispherical radiation properties of oxidized nickel at high temperatures, Corrosion Science 83 (2014) $272-280$.

[43] M. I. Mishchenko, L. D. Travis, Capabilities and limitations of a current Fortran implementation of the T-Matrix method for randomly oriented, rotationally symmetric scatters, Journal of Quantitative Spectroscopy and Radiative Transfer 60 (3) (1998) 309-324.

[44] M. I. Mishchenko, L. D. Travis, D. W. Mackowski, Extended precision Tmatrix code for randomly oriented nonspherical particles [cited April 18, 695 2016].

URL http://www.giss.nasa.gov/staff/mmishchenko/t_matrix.html

[45] A. Mugnai, W. J. Wiscombe, Scattering from nonspherical Chebyshev particles. I: cross sections, single-scattering albedo, asymmetry factor, and backscattered fraction, Applied Optics 25 (7) (1986) 1235-1244. 